\title{
Eldecalcitol, in Combination with Bisphosphonate, Is Effective for Treatment of Japanese Osteoporotic Patients
}

\author{
Keijiro Mukaiyama, ${ }^{1}$ Shigeharu Uchiyama, ${ }^{1}$ Yukio Nakamura, ${ }^{1}$ Shota Ikegami, ${ }^{1}$ \\ Akira Taguchi, ${ }^{2}$ Mikio Kamimura ${ }^{3}$ and Hiroyuki Kato ${ }^{1}$

\begin{abstract}
${ }^{1}$ Department of Orthopedic Surgery, Shinshu University School of Medicine, Matsumoto, Nagano, Japan
${ }^{2}$ Department of Oral and Maxillofacial Radiology, Matsumoto Dental University, Shiojiri, Nagano, Japan

${ }^{3}$ Center for Osteoporosis and Spinal Disorders, Kamimura Orthopaedic Clinic, Matsumoto, Nagano, Japan
\end{abstract}

\begin{abstract}
Alfacalcidol (ALF) and eldecalcitol (ELD) are vitamin D analogues that can be combined with anti-resorption drugs, such as bisphosphonate (BP) for the treatment of osteoporosis (OP). There has been no report comparing the effects of those vitamin $D$ analogs in combination with BPs. Twenty female patients with OP were enrolled, and all of them were treated with ALF and BPs. After switching from ALF to ELD, we examined the effectiveness of ALF and ELD. The averaged age was 69.4 years and the period of BP usage was between 1 to 13.4 years (mean period was 3.7 years). Serum corrected calcium, serum inorganic phosphorus, serum bone specific alkaline phosphatase (BAP), and serum tartrate-resistant acid phosphatase (TRACP)-5b were measured prior to ELD and at 6 months afterwards. Bone mineral density (BMD) of the lumbar spine (L-BMD), femoral neck, and total hip BMD were assessed one year before, prior to, and one year after ELD therapy commencement. Six months after switching from ALF to ELD, BAP and TRACP-5b values significantly decreased. After one year of ALF therapy, L-BMD, total hip BMD and femoral neck H-BMD values slightly increased. In contrast, a year following the change from ALF to ELD, L-BMD significantly increased and femoral neck BMD slightly increased, but total hip BMD did not. These results suggest that the treatment with ELD after ALF significantly suppressed bone turnover and increased L-BMD. Thus, the combined therapy with ELD is more effective for OP treatment than that with ALF.
\end{abstract}

Keywords : alfacalcidol; bisphosphonate; combination therapy; eldecalcitol; osteoporosis

Tohoku J. Exp. Med., 2015 December, 237 (4), 339-343. C 2015 Tohoku University Medical Press

\section{Introduction}

Vitamin D plays an important role in maintaining bone and calcium metabolism (Hegde et al. 2015). Vitamin D deficiency causes not only low bone mineral density (BMD) via secondary hyperparathyroidism, but also impairment of bone mineralization (Watanabe and Okazaki 2013).

At present, 25-hydroxyvitamin $\mathrm{D}_{3}, 25(\mathrm{OH}) \mathrm{D}_{3}$, is widely adopted in osteoporotic treatments, and administration of $25(\mathrm{OH}) \mathrm{D}_{3}$ is considered to be essential in clinical trials of osteoporosis (OP). Thus, evaluation of vitamin D status and its adequate provision are prerequisites for appropriate osteoporotic management and assessment.

$1 \alpha(\mathrm{OH})$ vitamin $\mathrm{D}_{3}$, alfacalcidol (ALF), is a vitamin $\mathrm{D}$ analogue (Fig. 1) that is frequently used for treatment of OP in Japan. ALF undergoes 25-hydroxylation in the liver, and $1,25(\mathrm{OH})_{2} \mathrm{D}_{3}$ thus formed promotes the absorption of calcium and phosphate (Orimo and Schacht 2005). Although ALF increases $1,25(\mathrm{OH})_{2} \mathrm{D}_{3}$ level and decreases serum parathyroid hormone, it does not affect bone turnover markers (Shiraki et al. 1999). Moreover, $1 \alpha, 25(\mathrm{OH})_{2}-$ $2 \beta$-(3-hydroxypropyloxy) $\mathrm{D}_{3}$, eldecalcitol (ELD), has been used for treatment of OP as a newly developed analog of $1,25(\mathrm{OH})_{2} \mathrm{D}_{3}$ (Fig. 1) (Hatakeyama et al. 2007; Hagino et al. 2015).

Bisphosphonate (BP) and selective estrogen receptor modulator (SERM) are representative anti-resorptive drugs (Miller et al. 2008; Itabashi et al. 2011) that are frequently combined with vitamin $\mathrm{D}$ analogues to treat osteoporotic patients. Combination therapy of oral BP and vitamin $\mathrm{D}$ analogues showed additive anti-osteoporotic effects as compared with BP monotherapy in postmenopausal women (Orimo et al. 2011; Sakai et al. 2015). Alendronate and ALF combination therapy reduced fracture risk, but only

Received August 19, 2015; revised and accepted October 29, 2015. Published online December 4, 2015; doi: 10.1620/tjem.237.339.

Correspondence: Yukio Nakamura, M.D., Ph.D., Department of Orthopaedic Surgery, Shinshu University School of Medicine, 3-1-1

Asahi, Matsumoto, Nagano 390-8621, Japan.

e-mail: yxn14@aol.jp

Shigeharu Uchiyama, M.D., Ph.D., Department of Orthopaedic Surgery, Shinshu University School of Medicine, 3-1-1 Asahi, Matsumoto, Nagano 390-8621, Japan.

e-mail: sigeuti@shinshu-u.ac.jp 
<smiles>C=C1CC[C@H](O)C/C1=C/C=C1\CCCC2C1CCC2C(C)CCCC(C)(C)O</smiles>

25(OH)D3

( Calcifediol)

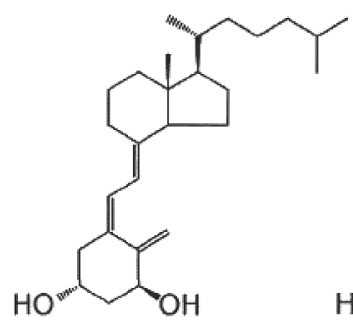

$1 \alpha(\mathrm{OH}) \mathrm{D} 3$

(Alfacalcidol)
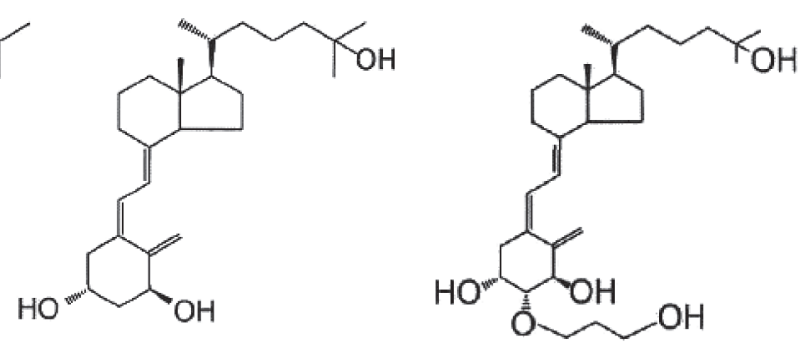

$1 \alpha, 25(\mathrm{OH}) 2 \mathrm{D} 3$

(Calcitriol)
1 $\alpha, 25(\mathrm{OH}) 2-2 \beta$-(3-hydroxypropyloxy)D3 ( Eldecalcitol)

Fig. 1. The structures of native vitamin D (calcifediol), active vitamin D (calcitriol), and vitamin D analogs (alfacalcidol and eldecalcitol).

during early treatment phases (Orimo et al. 2011). Moreover, the increased efficacy of combined alendronate and ELD treatment has been established over regimens containing, native vitamin D, and calcium in Japanese patients with primary OP. However, the combined ELD only increased the BMD in femoral neck regions (Sakai et al. 2015).

ELD has an inhibitory effect on bone resorption through the disruption of osteoclast formation. In a doubleblind randomized controlled trial of Japanese patients with OP, ELD was shown to increase BMD and decrease the incidence of vertebral fractures more effectively than ALF (Matsumoto et al. 2011). These results suggest that in the combination therapy of anti-resorptive drugs with vitamin D analogue, ELD is expected to have better effects on bone metabolism and BMD than ALF.

Randomized controlled trials (RCTs) are considered to provide the highest level of evidence. N-of- 1 studies also yield the superior analysis power when the subjects are the same, in which case statistical experts deem N-of-1 studies to be preferable (Cook et al. 1993; Gabler et al. 2011). However, there have been no investigations, such as $\mathrm{N}=1$ studies, comparing the effects of ELD and ALF in the same patients with respect to combination therapy with antiresorptive drugs.

Since there has been no report comparing the effects of these two vitamin D analogs in combination with other anti-resorptive agents, we analyzed the changes in bone turnover markers and BMD caused by ELD treatment concurrently prescribed with anti-resorptive drugs following ALF combination therapy in Japanese primary OP patients in the present study.

\section{Materials and Methods}

Twenty female patients with OP were enrolled whose treatment was ALF in combination with BPs. The combined drug was changed from ALF to ELD, then we examined the effectiveness of ALF and ELD at our institutions between April 2012 and April 2013. Only female patients were enrolled to avoid gender bias. The demographic patient data are summarized in Table 1. All of the vertebral fractures in this study were bone fragility fractures. All subjects were diagnosed as having primary OP in accordance with the revised criteria
Table 1. Background data of the 20 female patients.

\begin{tabular}{lc}
\hline \multicolumn{1}{c}{ Parameters } & Values \\
\hline Body height $(\mathrm{cm})$ & $150.0 \pm 5.5(142-162)$ \\
Body weight $(\mathrm{kg})$ & $48.4 \pm 6.2(34-58)$ \\
Age (years) & $69.4 \pm 11.0(34-84)$ \\
History of fracture prior to BP treatment & 8 cases $(+)$ \\
& 12 cases $(-)$ \\
Drug period (years) & $3.7 \pm 3.8(1-13.4)$ \\
\hline
\end{tabular}

Values are shown as mean \pm standard deviation and ranges where applicable.

established by the Japanese Society of Bone and Mineral Research (Soen et al. 2013).

Of the 20 patients receiving ALF $(1.0 \mu \mathrm{g}), 17$ were concomitantly administered BP (10 with alendronate and 7 with risedronate) and 3 were being given SERMs (bazedoxifene). Prior treatment with ALF and anti-resorptive drugs before ELD switching ranged from 1 to 13.4 years (average $3.7 \pm 3.8$ ). Each patient received $0.75 \mu \mathrm{g}$ ELD doses daily after breakfast.

Serum corrected calcium $(\mathrm{Ca})(\mathrm{mg} / \mathrm{dL})$ and inorganic phosphate (iP) $(\mathrm{mg} / \mathrm{dL})$ were measured for all subjects. Serum bone alkaline phosphatase (BAP) $(\mu \mathrm{g} / \mathrm{L})$ was evaluated as a marker of bone formation using a chemiluminescent enzyme immunoassay. Serum tartrateresistant acid phosphatase (TRACP)-5b (mU/dL) was assessed as a marker of bone resorption using the enzyme-linked immunosorbent assay. Serum intact parathyroid hormone (iPTH) $(\mathrm{pg} / \mathrm{mL})$ was measured by immunoradiometric assays. All serologic parameters were evaluated just prior to ELD administration and after 6 months of ELD treatment. Serum samples were collected between 8:30 a.m. and 10:00 a.m. after overnight fasting. Immunoassays were performed by SRL, Inc. (Tokyo, Japan).

BMD was measured using a dual-energy X-ray absorption fanbeam bone densitometer (Lunar Prodigy; GE Healthcare Bio-Sciences Corp., Piscataway, NJ, USA) at the L2-4 levels of the posteroanterior spine (L-BMD) and left hip. BMD values were obtained one year before, just prior to, and one year after commencing ELD therapy.

For statistical analysis, comparisons of markers at each measuring point were performed using paired $t$-test. BMD change was assessed with paired $t$-test with Bonferroni correction. $\alpha$ Error was designated as 0.05 . Statistical analyses were carried out using JPM 9 (SAS Institute Inc., USA). 
This study was approved by the institutional ethics review board (Protocol No. 2014-22) at Shinshu University School of Medicine prior to its start and was conducted in accordance with the ethical tenets outlined in the 1964 Declaration of Helsinki for research involving human subjects. Written informed consent was obtained from all patients.

\section{Results}

Before ALF regime conversion to ELD, enhanced bone metabolism was not evident with anti-resorptive drug therapy: mean TRACP-5b was $350.6 \pm 161.0 \mathrm{mU} / \mathrm{dL}$, BAP was $12.8 \pm 7.4 \mu \mathrm{g} / \mathrm{L}$, iPTH was $41.9 \pm 18.3 \mathrm{pg} / \mathrm{mL}$, corrected $\mathrm{Ca}$ was $9.47 \pm 0.4 \mathrm{mg} / \mathrm{dL}$, and $\mathrm{iP}$ was $3.42 \pm 0.47$ $\mathrm{mg} / \mathrm{dL}$, all of which were within normal ranges (Table 2).

Six months after switching from ALF to ELD, mean values for iPTH and iP were modestly decreased, and there was no change of $\mathrm{Ca}$ values. However, mean BAP was significantly decreased by $20.3 \%$ from $12.8 \pm 7.4$ to $10.2 \pm 3.3$ $(\mathrm{P}=0.049)$. TRACP-5b values were also significantly decreased from $350.6 \pm 161.0$ to $293.1 \pm 133.7(16.4 \%, \mathrm{P}=$ 0.005). These findings demonstrated that ELD combined with BP or SERM after ALF treatment resulted in marked decreases in bone turnover.

After a year of treatment with ALF combination therapy, L-BMD was marginally increased from $0.796 \pm 0.080$ to $0.801 \pm 0.084(\mathrm{P}=0.481)$, as were those of the femoral neck $(0.619 \pm 0.127$ to $0.638 \pm 0.103, \mathrm{P}=0.288)$ and total hip $(0.663 \pm 0.100$ to $0.668 \pm 0.103, \mathrm{P}=0.105)$, which corresponded to BMD gains of $0.63 \%, 0.3 \%$, and $0.8 \%$, respectively (Table 3). Thus, hip and lumbar BMD were not remarkably affected by ALF combined with of BP or SERM in the year prior to the switching.

Twelve months after the conversion to bone resorption inhibitors plus ELD therapy, total hip BMD values were largely unchanged $(\mathrm{P}=0.959)$ (Table 4$)$. Femoral neck BMD values were increased $(0.638 \pm 0.103$ to $0.648 \pm$ $0.107 ; 1.7 \%)$, albeit not significantly $(\mathrm{P}=0.132)$. Most notably, L-BMD was significantly increased from $0.801 \pm$ 0.084 to $0.819 \pm 0.086$, a gain of $2.2 \%(\mathrm{P}=0.0097)$.

\section{Discussion}

The results of the current study demonstrated that 1) bone metabolism was already being modulated ahead of ELD treatment by long-term administration of anti-resorptive drugs, such as BP, and ALF, 2) bone formation (BAP) and bone resorption (TRACP-5b) markers were significantly reduced by ELD treatment with concurrently administered BP or SERM, and 3) ELD in place of ALF therapy did not markedly affect hip BMD but significantly increased L-BMD.

ELD is an analogue of 1,25-dihydroxyvitamin $\mathrm{D}_{3}$ that has been shown to improve BMD. ELD is more efficacious than ALF in preventing vertebral and wrist fractures in osteoporotic patients with vitamin D insufficiency, but with

Table 2. Changes in serum bone metabolic markers after switching from ALF to ELD.

\begin{tabular}{lccc}
\hline & At the time of switching to ELD & Six months after switching to ELD & P value \\
\hline BAP $(\mu \mathrm{g} / \mathrm{L})$ & $12.8 \pm 7.4$ & $10.2 \pm 3.3$ & $0.049 *$ \\
TRACP-5b $(\mathrm{mU} / \mathrm{dL})$ & $350.6 \pm 161.0$ & $293.1 \pm 133.7$ & $0.005^{* *}$ \\
$\mathrm{iPTH}(\mathrm{pg} / \mathrm{mL})$ & $41.9 \pm 18.3$ & $39.9 \pm 15.3$ & 0.37 \\
$\mathrm{Ca}(\mathrm{mg} / \mathrm{dL})$ & $9.47 \pm 0.4$ & $9.50 \pm 0.43$ & 0.69 \\
$\mathrm{iP}(\mathrm{mg} / \mathrm{dL})$ & $3.42 \pm 0.47$ & $3.30 \pm 0.41$ & 0.30 \\
\hline
\end{tabular}

Values are shown as mean \pm standard deviation. Asterisks indicate significant difference.

Table 3. Changes in BMD values during the year prior to switching from ALF to ELD.

\begin{tabular}{lcccc}
\hline & One year prior to switching to ELD & At the time of switching to ELD & P value & Ratio of Increase \\
\hline L2-4 BMD & $0.796 \pm 0.080$ & $0.801 \pm 0.084$ & 0.481 & $0.63 \%$ \\
Femoral neck BMD & $0.636 \pm 0.106$ & $0.638 \pm 0.103$ & 0.670 & $0.31 \%$ \\
Total hip BMD & $0.663 \pm 0.100$ & $0.668 \pm 0.103$ & 0.105 & $0.75 \%$ \\
\hline
\end{tabular}

Values are shown as mean \pm standard deviation.

Table 4. Changes in BMD values during the year after switching from ALF to ELD.

\begin{tabular}{lcccc}
\hline & At the time of switching to ELD & One year after switching to ELD & P value & Ratio of Increase \\
\hline L2-4 BMD & $0.801 \pm 0.084$ & $0.819 \pm 0.086$ & 0.0097 & $2.25 \%$ \\
Femoral neck BMD & $0.638 \pm 0.103$ & $0.649 \pm 0.107$ & 0.132 & $1.72 \%$ \\
Total hip BMD & $0.668 \pm 0.103$ & $0.669 \pm 0.108$ & 0.959 & $0.15 \%$ \\
\hline
\end{tabular}

Values are shown as mean \pm standard deviation. 
a safety profile similar to that of ALF. Specifically, ELD therapy reduced the frequency of wrist fracture by approximately $71 \%$ over a 144 -week treatment period versus an ALF group (Matsumoto et al. 2011), and ELD did not affect serum $25(\mathrm{OH}) \mathrm{D}_{3}$ levels, reduced the incidence of osteoporotic fracture, and increased BMD as compared with ALF, regardless of serum $25(\mathrm{OH}) \mathrm{D}_{3}$ concentrations of $20 \mathrm{ng} / \mathrm{mL}$ or more (Matsumoto and Kubodera 2007).

Sakai et al. (2015) recently reported values of bone turnover markers being significantly lower in alendronate plus ELD group than in a group maintained with BP and native vitamin D. However, the difference between the groups was not significant. They also uncovered that serum BAP and TRACP-5b were significantly reduced, albeit by a modest 20\% (Sakai et al. 2015). As reported in a phase III study, ELD exerts inhibitory effects on bone metabolism (Matsumoto et al. 2011). Together with the fact that ALF does not affect bone turnover markers (Shiraki et al. 1999). Our findings indicate the additive effects of BP- and ELDmediated inhibition of bone turnover.

An earlier longitudinal analysis of hip geometry by clinical computed tomography revealed a potential role of ELD in increasing cortical cross-sectional area, BMD, and bone mass (Ito et al. 2011). The authors also suggest that by improving the biomechanical properties of the proximal femur, ELD may reduce the risk of hip fractures (Ito et al. 2011).

Even after a regimen change to ELD, total hip BMD values in the present study did not increase, while femoral neck BMD rose by $1.7 \%$. Sakai et al. (2015) reported that femoral neck BMD values only were increased in a BP and ELD group in comparison with a BP and vitamin D group. In our investigation, total hip BMD did not increase with ELD but L-BMD values were significantly augmented. The reason for this discrepancy requires further study.

RCTs are considered to provide a high level of evidence since there are no perfectly matched groups in research studies due to variations in environmental and genetic factors. However, as N-of-1 studies evaluate identical subjects, they require fewer participants and can be regarded as a kind of RCT since the intervention for each subject is open-labeled and randomized (Cook et al. 1993; Gabler et al. 2011). N-of-1 studies necessitate certain of wash-out period to return to baseline levels between interventions (Cook et al. 1993; Gabler et al. 2011). Thus, our current study is not considered as an N-of-1 study; namely, there might have been synergistic effects between ALF and ELD. However, for reasons that 1) the half-life of ALF is very short at 17.6 hours (Tohira et al. 1979) and 2) BMD values in the total hip did not increase by ELD treatment but were raised slightly by ALF therapy, which suggested the absence of additive ALF effects.

Lastly, the order of drug administration was not randomized in this trial. The effectiveness of BP on BMD diminishes over long treatment periods (Bone et al. 2004). Itabashi et al. (2011) recently described that SERM signifi- cantly increased BMD for up to 104 weeks after treatment, although increasing ratio plateaued or worsened slightly after a few years of treatment. Thus, the effects on BMD of BP and SERM presumably decreased over time, which may have favored the assessment of the firstly administered ALF.

In this study, one of the limitations is that there was a broad range of the anti-resorptive drug treatment period. Although the shorter BP pre-treatment made the effectiveness of ELD after ALF treatment disadvantage, the ELD treatment significantly increased BMD values in this study. Thus, we believe that the variety of the administered period has not affected our results. However, significant gains in lumbar BMD were seen for ELD, which confirmed that the drug exerted additional effects on BMD than did ALF.

In conclusion, ELD after switching from ALF significantly inhibited bone turnover and increased lumbar BMD in patients with primary OP. In the combined therapy of BP or SERM with a vitamin D analogue, ELD would be more effective than ALF with respect to the lumbar BMD increase. Larger randomized trials of ELD combination treatment are warranted.

\section{Authors' Contribution}

Y.N. and S.U. directed this study. K.M. and M.K. collected the patients' data and prepared all of the tables. Y.N., K.M., and M.K. wrote the main manuscript text. K.M. and S.I. performed statistical analyses. A.T. and H.K. gave suggestions on this study. All authors reviewed the manuscript.

\section{Conflict of Interest}

The authors declare no conflict of interest.

\section{References}

Bone, H.G., Hosking, D., Devogelaer, J.P., Tucci, J.R., Emkey, R.D., Tonino, R.P., Rodriguez-Portales, J.A., Downs, R.W., Gupta, J., Santora, A.C. \& Liberman, U.A.; Alendronate Phase III Osteoporosis Treatment Study Group (2004) Ten years' experience with alendronate for osteoporosis in postmenopausal women. N. Engl. J. Med., 350, 1189-1199.

Cook, D.J., Guyatt, G.H., Davis, C., Willan, A. \& McIlroy, W. (1993) A diagnostic and therapeutic N-of-1 randomized trial. Can. J. Psychiatry, 38, 251-254.

Gabler, N.B., Duan, N., Vohra, S. \& Kravitz, R.L. (2011) N-of-1 trials in the medical literature: a systematic review. Med. Care, 49, 761-768.

Hagino, H. (2015) Vitamin D3 analogs for the treatment of osteoporosis. Can. J. Physiol. Pharmacol., 93, 327-332.

Hatakeyama, S., Nagashima, S., Imai, N., Takahashi, K., Ishihara, J., Sugita, A., Nihei, T., Saito, H., Takahashi, F. \& Kubodera, N. (2007) Synthesis and biological evaluation of a 3-positon epimer of 1alpha,25-dihydroxy-2beta-(3-hydroxypropoxy) vitamin D3 (ED-71). J. Steroid Biochem. Mol. Biol., 103, 222-226.

Hegde, V., Jo, J.E., Andreopoulou, P. \& Lane, J.M. (2015) Effect of osteoporosis medications on fracture healing. Osteoporos. Int., doi: 10.1007/s00198-015-3331-7. [Epub ahead of print].

Itabashi, A., Yoh, K., Chines, A.A., Miki, T., Takada, M., Sato, H., Gorai, I., Sugimoto, T., Mizunuma, H., Ochi, H., Constantine, G.D. \& Ohta, H. (2011) Effects of bazedoxifene on bone mineral density, bone turnover, and safety in postmenopausal 
Japanese women with osteoporosis. J. Bone Miner. Res., 26, 519-529.

Ito, M., Nakamura, T., Fukunaga, M., Shiraki, M. \& Matsumoto, T. (2011) Effect of eldecalcitol, an active vitamin D analog, on hip structure and biomechanical properties: 3D assessment by clinical CT. Bone, 49, 328-334.

Matsumoto, T., Ito, M., Hayashi, Y., Hirota, T., Tanigawara, Y., Sone, T., Fukunaga, M., Shiraki, M. \& Nakamura, T. (2011) A new active vitamin D3 analog, eldecalcitol, prevents the risk of osteoporotic fractures: a randomized, active comparator, double-blind study. Bone, 49, 605-612.

Matsumoto, T. \& Kubodera, N. (2007) ED-71, a new active vitamin $\mathrm{D} 3$, increases bone mineral density regardless of serum 25(OH)D levels in osteoporotic subjects. J. Steroid Biochem. Mol. Biol., 103, 584-586.

Miller, P.D., Chines, A.A., Christiansen, C., Hoeck, H.C., Kendler, D.L., Lewiecki, E.M., Woodson, G., Levine, A.B., Constantine, G. \& Delmas, P.D. (2008) Effects of bazedoxifene on BMD and bone turnover in postmenopausal women: 2-yr results of a randomized, double-blind, placebo-, and active-controlled study. J. Bone Miner. Res., 23, 525-535.

Orimo, H., Nakamura, T., Fukunaga, M., Ohta, H., Hosoi, T., Uemura, Y., Kuroda, T., Miyakawa, N., Ohashi, Y. \& Shiraki, M.; A-TOP (Adequate Treatment of Osteoporosis) research group (2011) Effects of alendronate plus alfacalcidol in osteoporosis patients with a high risk of fracture: the Japanese Osteoporosis Intervention Trial (JOINT) - 02. Curr. Med. Res. Opin., 27, 1273-1284.

Orimo, H. \& Schacht, E. (2005) The D-hormone analog alfacalcidol: the pioneer beyond the horizon of osteoporosis treatment. J. Rheumatol. Suppl., 76, 4-10.

Sakai, A., Ito, M., Tomomitsu, T., Tsurukami, H., Ikeda, S.,
Fukuda, F., Mizunuma, H., Inoue, T., Saito, H. \& Nakamura, T.; e-ADVANCED Study Group (2015) Efficacy of combined treatment with alendronate (ALN) and eldecalcitol, a new active vitamin D analog, compared to that of concomitant ALN, vitamin D plus calcium treatment in Japanese patients with primary osteoporosis. Osteoporos. Int., 26, 1193-1202.

Shiraki, M., Kushida, K., Fukunaga, M., Kishimoto, H., Taga, M., Nakamura, T., Kaneda, K., Minaguchi, H., Inoue, T., Morii, H., Tomita, A., Yamamoto, K., Nagata, Y., Nakashima, M. \& Orimo, H. (1999) A double-masked multicenter comparative study between alendronate and alfacalcidol in Japanese patients with osteoporosis. The Alendronate Phase III Osteoporosis Treatment Research Group. Osteoporos. Int., 10, 183-192.

Soen, S., Fukunaga, M., Sugimoto, T., Sone, T., Fujiwara, S., Endo, N., Gorai, I., Shiraki, M., Hagino, H., Hosoi, T., Ohta, H., Yoneda, T. \& Tomomitsu, T.; Japanese Society for Bone and Mineral Research and Japan Osteoporosis Society Joint Review Committee for the Revision of the Diagnostic Criteria for Primary Osteoporosis (2013) Diagnostic criteria for primary osteoporosis: year 2012 revision. J. Bone Miner. Metab., 31, 247-257.

Tohira, Y., Nakano, H., Nanjo, M., Kumaki, K., Takanashi, S., Nishii, Y. \& Suda, T. (1979) Determination of 1 alpha,25$(\mathrm{OH}) 2-\mathrm{D}$ in rat and human plasma by radioreceptor assay following oral administration of 1 alpha-OH-D3. Bone Metabolism, 12, 152-162 (in Japanese).

Watanabe, R. \& Okazaki, R. (2013) Secondary osteoporosis or secondary contributors to bone loss in fracture. Vitamin D deficiency and fracture. Clin. Calcium, 23, 1313-1319 (in Japanese). 